Marco Vargas ${ }^{1,11}$

Carlos Augusto Grabois

Gadelha" IIIII,IV

Laís Silveira Costa"

José Maldonado ${ }^{\mathrm{II}, \mathrm{IV}}$

\section{Inovação na indústria química e biotecnológica em saúde: em busca de uma agenda virtuosa}

Departamento de Economia. Faculdade de Economia. Universidade Federal Fluminense. Niterói, RJ, Brasil

Grupo de Pesquisa de Inovação em Saúde. Escola Nacional de Saúde Pública Sergio Arouca (ENSP). Fundação Oswaldo Cruz (Fiocruz). Rio de Janeiro, RJ, Brasil

III Secretaria de Ciência, Tecnologia e Insumos Estratégicos. Ministério da Saúde. Brasília, DF, Brasil

Mestrado Profissional em Política e Gestão de CT\&I em Saúde. ENSP-Fiocruz. Rio de Janeiro, RJ, Brasil

\section{Correspondência | Correspondence: Marco Vargas \\ Rua Tirandentes, 17 - Ingá \\ 24210-510 Niterói, RJ, Brasil \\ E-mail: mvargas@economia.uff.br}

\section{Recebido: 16/2/2012}

Aprovado: 19/9/2012

Artigo disponível em português e inglês em: www.scielo.br/rsp

\section{Innovation in pharmaceutical and health biotechnology industries: challenges for a virtuous agenda}

\section{RESUMO}

As indústrias de base química e biotecnológica compõem um dos subsistemas produtivos do Complexo Produtivo da Saúde, que se destaca tanto pela sua relevância econômica como pela sua importância no domínio de novas tecnologias em áreas estratégicas. A consolidação do segmento de produção de medicamentos genéricos na última década proporcionou um aumento significativo da participação de empresas nacionais no mercado farmacêutico e representou um importante ponto de inflexão na trajetória de crescimento do setor. Entretanto, ainda há importantes gargalos estruturais tanto na base produtiva como em termos de seu dinamismo inovativo. Tais gargalos revelam a elevada vulnerabilidade do Sistema Nacional de Saúde e apontam para a importância da criação e implementação de políticas públicas que articulem o desenvolvimento da base produtiva e da inovação na indústria farmacêutica com o atendimento das demandas sociais relativas à saúde no País.

DESCRITORES: Biotecnologia, economia. Indústria Química, economia. Desenvolvimento Tecnológico, políticas. Política Nacional de Ciência, Tecnologia e Inovação.

\section{ABSTRACT}

Pharmaceutical and biotechnology industries comprise a major production subsystem of the health industrial complex in Brazil. It stands out for both its economic importance and its prominent role in developing new technologies in strategic areas. Strengthening the local production of generic drugs in the last decade has significantly increased the number of Brazilian companies in the local pharmaceutical market and has been an important turning point for this industry's growth. However, there remain major structural bottlenecks both in terms of production and continuous innovation. These bottlenecks reveal the high vulnerability of the Brazilian National Health System and point to the need of public policies that promote strengthening the production base and innovation in the pharmaceutical industry and that at the same time meet health-related social demands in health in Brazil.

DESCRIPTORS: Biotechnology, economics. Chemical Industry, economics. Technological Development, policies. National Science, Technology and Innovation Policy. 


\section{INTRODUÇÃO}

A consolidação da produção de medicamentos genéricos no Brasil na década de 2000 proporcionou um aumento da participação de empresas nacionais no mercado farmacêutico e representou um importante ponto de inflexão na trajetória de crescimento da indústria farmacêutica nacional. Entretanto, alguns fatores mostram a necessidade de mudanças estruturais profundas na indústria de base química e biotecnológica no País, a saber: aumento das pressões competitivas no segmento de genéricos, recente realinhamento da estratégia corporativa dos grandes laboratórios multinacionais e fragilidade da base produtiva. Tais mudanças envolvem não somente um esforço de adensamento da cadeia produtiva farmacêutica como também a adoção de estratégias de inovação robustas para a produção nacional de produtos com maior valor agregado, particularmente por meio de plataformas tecnológicas baseadas nos avanços da biotecnologia.

Este artigo apresenta um breve panorama sobre as tendências e desafios que marcam a trajetória recente da indústria de base química e biotecnológica em saúde no Brasil. Além disso, a crescente articulação entre as políticas públicas na área da saúde e no campo industrial e tecnológico tem permitido conciliar o aumento na capacidade produtiva e de inovação dessa indústria com o atendimento das demandas sociais relativas à saúde no País.

\section{INDÚSTRIA DE BASE QUÍMICA E BIOTECNOLÓGICA EM SAÚDE NO BRASIL}

Em 2011, o mercado farmacêutico brasileiro ocupava a oitava posição no ranking internacional de vendas globais da indústria farmacêutica e contava com um faturamento da ordem de R $\$ 43$ bilhões. ${ }^{\mathrm{a}}$ Apesar do claro predomínio das grandes empresas multinacionais que dominam o mercado nacional em diferentes segmentos e classes terapêuticas, houve aumento na participação de empresas nacionais no mercado ao longo da década de 2000. Estima-se que a participação das empresas de capital nacional no total do mercado farmacêutico brasileiro entre 2003 e 2010 tenha aumentado de 32,5\% para mais de 50\%. Em 2010, quatro laboratórios farmacêuticos nacionais - Aché, EMS, Eurofarma e Hypermarcas - figuravam entre as dez maiores empresas do setor no País, enquanto no passado essa presença se limitava a uma ou duas empresas. ${ }^{4}$

Houve, portanto, uma mudança estrutural no contexto nacional em termos de capacidade produtiva de medicamentos finais formulados que resultou num aumento da participação de empresas nacionais no mercado brasileiro. Tal fenômeno esteve particularmente associado à consolidação do mercado de medicamentos genéricos. Entretanto, as empresas nacionais ainda apresentam porte reduzido comparativamente aos conglomerados multinacionais e uma inserção reduzida em nichos de produtos de maior valor agregado, em particular no caso de fármacos e medicamentos produzidos a partir de rotas biotecnológicas. ${ }^{3,4} \mathrm{Em}$ termos gerais, o panorama atual da indústria de base química e biotecnológica em saúde no Brasil pode ser sintetizado a partir dos seguintes pontos: ${ }^{\mathrm{b}}$

- o Complexo Econômico Industrial da Saúde (CEIS) como um todo e a indústria de base química e biotecnológica em particular contam com elevada participação relativa no produto interno bruto, no valor agregado industrial e no emprego qualificado do Brasil, mas também respondem por uma participação elevada no déficit da balança comercial do País; ${ }^{2}$

- a análise da estrutura produtiva ainda revela importantes gargalos estruturais na cadeia produtiva, sobretudo na produção de fármacos (insumos farmacêuticos ativos);

- a retomada recente no crescimento do setor farmacêutico, fomentada pela expansão do mercado de genéricos, viabilizou o fortalecimento das empresas farmacêuticas nacionais, mas também tem constituído um forte estímulo para a entrada dos grandes laboratórios farmacêuticos multinacionais no mercado brasileiro por meio da aquisição de empresas locais;

- as empresas do setor farmacêutico no Brasil ainda apresentam investimentos reduzidos em atividades inovativas e de pesquisa e desenvolvimento (P\&D), tanto em termos do padrão internacional da indústria farmacêutica como em relação aos setores mais dinâmicos da indústria brasileira;

- a estrutura produtiva em saúde no Brasil conta com o papel destacado desempenhado pelos laboratórios oficiais na Política Nacional de Saúde, na produção de medicamentos para o Sistema Único de Saúde (SUS), no suporte à regulação ou no processo de ampliação da capacitação tecnológica nacional;

- elevada concentração da estrutura produtiva da indústria farmacêutica nas regiões Sul e Sudeste, particularmente nos estados de São Paulo, Rio de Janeiro e Minas Gerais;

- forte assimetria da concentração da estrutura produtiva industrial na distribuição da infraestrutura cientifica e tecnológica ligada ao esforço de pesquisa, desenvolvimento e inovação em saúde.

\footnotetext{
a Sindicato da Indústria de Produtos Farmacêuticos no Estado de São Paulo - Sindusfarma. Indicadores econômicos. São Paulo. [citado 2012 out 12]. Disponível em: http://www.sindusfarmacomunica.org.br/indicadores-economicos/

${ }^{\text {b}}$ Vargas MA, Gadelha C, Maldonado J, Barbosa P. Reestruturação na indústria farmacêutica mundial e seus impactos na dinâmica produtiva e inovativa do setor farmacêutico brasileiro. In: XV Encontro Nacional de Economia Política; 2010; São Luis do Maranhão, BR [citado 2012 out 12]. Disponível em: http://www.sep.org.br/artigos?conference=15\&title=\&author=vargas
} 
Um dos principais reflexos da fragilidade da produção em saúde no âmbito da indústria de base química e biotecnológica no Brasil reside no crescimento acelerado do déficit na balança comercial de fármacos e medicamentos desde os anos 2000. Apesar do dinamismo recente do mercado farmacêutico brasileiro, houve uma expansão acelerada das importações de fármacos e medicamentos. Tal fato resultou em desequilíbrio estrutural da balança comercial, particularmente nos segmentos mais intensivos em conhecimento da indústria farmacêutica.

Análise da evolução da balança comercial para o conjunto de segmentos da indústria de base química e biotecnológica entre 1996 e 2011 mostra a aceleração no montante de importações a partir da segunda metade da década 2000, conforme ilustra a Figura. Essa foi uma tendência comum a todos os segmentos da indústria de base química e biotecnológica, resultando num aumento significativo do déficit na balança comercial, particularmente nos segmentos de fármacos, medicamentos e hemoderivados. De acordo com dados do Grupo de Pesquisa de Inovação em Saúde da Fiocruz, entre 2005 e 2011, as importações para o conjunto de segmentos da indústria de base química e tecnológica passaram de US \$ 1,7 bilhão para US\$ 3,7 bilhões, um crescimento de $121 \%$ em seis anos.

A importação de produtos de base biotecnológica tem apresentado uma forte contribuição no aumento exponencial do déficit na balança de fármacos e medicamentos. ${ }^{c}$ Estima-se que, entre 2005 e 2010, as importações de produtos biológicos tenham crescido em média 37\% ao ano. Da mesma forma, oito entre os dez principais medicamentos com maior valor de importação são produzidos por rota biotecnológica. ${ }^{3}$

Ainda que os produtos farmacêuticos oriundos de síntese química respondam pela maior parte das receitas da indústria farmacêutica, os de origem biotecnológica estão entre os medicamentos mais vendidos no mundo, cuja participação relativa no mercado farmacêutico global tem crescido expressivamente. ${ }^{1}$ Estima-se que até 2016 os produtos de origem biotecnológica deverão responder por aproximadamente $45 \%$ das vendas entre os 100 principais produtos farmacêuticos no mercado global, ou o equivalente a US\$ 192 bilhões. A convergência entre essas diferentes plataformas tecnológicas ligadas à biologia molecular (genômica, proteômica e outros), bioinformática, nanobiotecnologia, ciências de materiais e outros representa uma tendência global e deve servir de parâmetro para promoção da mudança estrutural nos diversos segmentos da indústria de base química e biotecnológica em saúde no Brasil.

Em termos da infraestrutura científica e tecnológica, a maior parte dos institutos públicos de pesquisa do Brasil conta com grupos consolidados de pesquisa na área da
US\$ Milhões

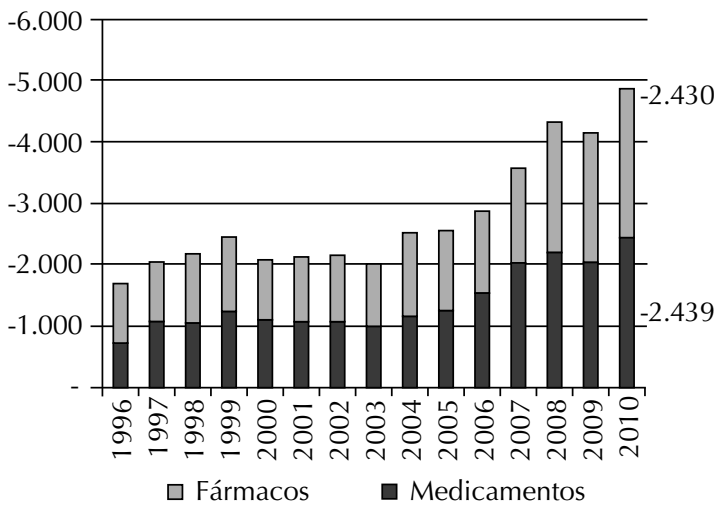

Fonte: Dados fornecidos pelo Grupo de Inovação em Saúde da Fiocruz em 2012, com base em dados do sistema Alice do Ministério do Desenvolvimento, Indústria e Comércio Exterior.

Figura. Déficit na balança comercial de fármacos e medicamentos, 1996-2010.

saúde e em algumas áreas de fronteira da biotecnologia aplicada à saúde. Entretanto, na busca de oportunidades para a inserção brasileira em nichos estratégicos, há questões críticas a serem equacionadas; por exemplo, o descompasso entre o elevado grau de capacitação científica em diferentes áreas da biotecnologia e a limitada capacitação inovativa existente hoje na base produtiva em saúde.

A existência desse quadro traz importantes implicações, seja em termos da vulnerabilidade que acarreta para o sistema nacional de saúde, seja pelo risco de que a base produtiva em saúde no Brasil se acomode a um padrão pouco inovador. Torna-se fundamental, portanto, a análise de alternativas em termos de políticas públicas que possam contribuir para um cenário de ruptura voltado ao desenvolvimento da indústria de base química e biotecnológica em saúde no País.

\section{CONSIDERAÇÕES FINAIS}

Diante desse novo cenário a ação reguladora do Estado torna-se crucial para viabilizar o aproveitamento de novas janelas de oportunidade que permitam articular o desenvolvimento da indústria de base química e biotecnológica em saúde com a política de saúde e com a demanda de bem-estar social. Apesar das dificuldades de implementação e de coordenação de instrumentos e ações para essa área, percebe-se no momento atual um movimento promissor de convergência da Política Industrial e Tecnológica com a Política de Saúde. ${ }^{3}$

Dentre as novas diretrizes que colocam a saúde como espaço estratégico de política destaca-se, em particular,

c Gadelha CAG. Biotecnologia em saúde: um estudo da mudança tecnológica na indústria farmacêutica e das perspectivas de seu desenvolvimento no Brasil [dissertação de mestrado]. Campinas: Instituto de Economia da Unicamp; 1990. 
o uso do poder de compra do Estado, o aprimoramento do marco regulatório com vistas ao apoio à inovação e o estabelecimento de novos instrumentos de financiamento ao setor produtivo. Ainda que a análise detalhada desses instrumentos extrapole os objetivos deste ensaio, é possível apontar para as linhas gerais de orientação das políticas de apoio ao desenvolvimento do complexo da saúde cujas ações de coordenação e articulação de políticas vêm sendo desempenhadas pelo Grupo Executivo do Complexo Industrial da Saúde.

No tocante ao uso do poder de compra é possível destacar um conjunto de medidas e instrumentos que buscam aliar a consolidação da base produtiva em saúde com o desenvolvimento de produtos com maior valor agregado e conteúdo tecnológico. Tais medidas incluem a publicação da lista de produtos prioritários para o SUS (Portaria 1.284, de 2010 ), a nova Lei de Compras Públicas, ${ }^{\mathrm{e}}$ que contempla uma margem de preferência para fármacos e medicamentos estratégicos produzidos no Brasil, e as Parcerias para Desenvolvimento Produtivo (PDP) envolvendo laboratórios oficiais e privados. A lista de produtos prioritários sinaliza quais deles constituem foco prioritário para o aumento da produção local, seja pela elevada importância social e econômica, pela sua relevância na busca de novas capacitações tecnológicas na área da saúde. ${ }^{\text {f A regula- }}$ mentação da Lei 12.349 (Lei de Compras Públicas), por sua vez, visa utilizar de forma escalonada uma margem de preferência (de no máximo 25\%) para licitação de produtos manufaturados e serviços nacionais resultantes de desenvolvimento e inovações tecnológicas realizadas no País. Finalmente, as PDP visam prioritariamente internalizar a tecnologia de produção de fármacos e medicamentos estratégicos nos laboratórios públicos por meio de processos de transferência de tecnologia.

No campo regulatório, observa-se um movimento importante de busca de uma maior articulação e conciliação entre a lógica sanitária (que implica padrões de segurança técnica e equilíbrio econômico no sistema) e as necessidades de fomentar a dinâmica de inovação e competitividade no segmento de fármacos e medicamentos. Tal movimento se reflete no avanço normativo de instituições como a Agência Nacional de Vigilância Sanitária, no sentido de incorporar demandas do setor industrial que visam à adequação do marco regulatório.

Finalmente, no âmbito das ações de incentivo e financiamento destaca-se a importância crescente de programas de apoio ao CEIS que contemplam tanto a expansão da capacidade produtiva no setor farmacêutico como a consolidação de competências para inovar. No primeiro caso, menciona-se o Programa de Apoio ao Desenvolvimento da Cadeia Produtiva Farmacêutica do Banco Nacional de Desenvolvimento. No caso das ações de incentivo no campo da ciência, tecnologia e inovação, cabe destacar o papel da Financiadora de Projetos por meio dos Fundos Setoriais, como o CT-Saúde e o CT-Bio, cujos objetivos envolvem o processo de capacitação tecnológica nas áreas de interesse do SUS e o estímulo ao aumento dos investimentos privados em P\&D na área da saúde.

\section{REFERÊNCIAS}

1. Evaluate Pharma. World preview 2016: beyond the Patent Cliff. London; 2011.

2. Gadelha CAG. O complexo industrial da saúde e a necessidade de um enfoque dinâmico na economia da saúde. Cienc Saude Coletiva. 2003;8(2):521-35. DOI:10.1590/S1413-81232003000200015

3. Gadelha CAG, Maldonado JMSV, Vargas MA, Barbosa

P, Costa LS. A dinâmica do sistema produtivo da saúde: inovação e complexo econômico-industrial. Rio de Janeiro: Editora Fiocruz; 2012. No prelo.

4. Reis C, Landin A, Pieroni JP. Lições da experiência internacional e proposta para incorporação da rota biotecnológica na Indústria farmacêutica brasileira. Rio de Janeiro: BNDES; 2011. p.5-44. (BNDES Setorial, 34).

O Grupo de Inovação em Saúde conta com apoio sistemático da Organização Pan Americana de Saúde (Contrato nº ENSP043-CAC-12).

Artigo submetido ao processo de julgamento por pares adotado para qualquer outro manuscrito submetido a este periódico, com anonimato garantido entre autores e revisores. Editores e revisores declaram não haver conflito de interesses que pudesse afetar o processo de julgamento do artigo.

Os autores declaram não haver conflito de interesses.

\footnotetext{
d Ministério da Saúde. Portaria n 1.284, de 26 de maio de 2010. Altera o anexo da Portaria n 978/GM/MS, de 16 de maio de 2008 [citado 2012 out 12]. Disponível em: http://portal.saude.gov.br/portal/arquivos/pdf/portaria1284_070710.pdf

e A Medida Provisória ${ }^{\circ}$ 495, de 19 de julho de 2010, posteriormente convertida na Lei n ${ }^{\circ} 12.349$, de 15 de dezembro de 2010 alterou a Lei $8.666 / 93$ concedendo margens de preferência de até $25 \%$ aos produtos brasileiros nas licitações públicas.

${ }^{\mathrm{f}}$ Ministério da Saúde, Gabinete do Ministro. Portaria n 978 de 16 de maio de 2008. Dispõe sobre a lista de produtos estratégicos, no âmbito do Sistema Único de Saúde, com a finalidade de colaborar com o desenvolvimento do Complexo Industrial da Saúde e institui a Comissão para Revisão e Atualização da Referida Lista [citado 2012 out 12]. Disponível em: http://bvsms.saude.gov.br/bvs/saudelegis/gm/2008/ prt0978_16_05_2008.html
} 